\title{
LETTER
}

\section{Circulating neutrophil counts and mortality in septic shock}

\author{
Jesús F Bermejo-Martín ${ }^{1 *}$, Eduardo Tamayo ${ }^{2 \dagger}$, Gema Ruiz ${ }^{3 \dagger}$, David Andaluz-Ojeda ${ }^{4 \dagger}$, Rubén Herrán-Monge ${ }^{5 \dagger}$, \\ Arturo Muriel-Bombín ${ }^{5}$, Maria Fe Muñoz ${ }^{1}$, María Heredia-Rodríguez², Rafael Citores ${ }^{4}$, José Ignacio Gómez-Herreras ${ }^{2}$, \\ Jesús Blanco ${ }^{5,6+}$ and for the EXPRESS (Expresión Génica en Sepsis) and GRECIA (Grupo de Estudios y Análisis en \\ Cuidados Intensivos) groups
}

Polynuclear neutrophils can play dual roles in sepsis: on the one hand they mediate major antimicrobial activities and on the other hand they can contribute to the development of multiple organ failure [1]. Nonetheless, in spite of the importance of these cells in sepsis, the influence of the circulating neutrophil count $(\mathrm{CNC})$ on the prognosis of septic patients with this pathology has not been properly evaluated.

We analyzed the association between $\mathrm{CNC}$ and outcome in two cohorts of patients with diagnostic criteria of septic shock (SS) [2]: the first was recruited in the context of a single center study (EXPRESS study, discovery cohort, $\mathrm{n}=195$; Table 1 ), and the second in the context of a multi-centric study (GRECIA study, validation cohort, $\mathrm{n}=194$; Table 2). Written informed consent was obtained from each patient or their legal representative. The two studies were approved by the Research Ethics Committee of the Hospital Clínico Universitario, Valladolid, Spain (for the EXPRESS study) and Hospital Universitario Río Hortega, Valladolid, Spain (coordinating center for the GRECIA study).

When patients of the discovery cohort were split based on deciles for $\mathrm{CNC}$ at SS diagnosis, those with $\mathrm{CNC}$ $<7,226$ cells $/ \mathrm{mm}^{3}$ (decile 2) died earlier than the other non-survivors (Figure 1). Multivariate Cox regression analysis showed that patients with $\mathrm{CNC}$ below this cutoff value had an almost two-fold risk of death (Figure 1). The cutoff value was evaluated again in the validation cohort, with similar results (Figure 1). Counts of other leukocyte subtypes had no significant association with outcome.
Although normal reference values in blood vary depending on sex, race and age, available literature supports that 7,226 cells $/ \mathrm{mm}^{3}$ is at the upper limit of normal $\mathrm{CNC}$ values [3]. Patients with insufficient numbers of circulating neutrophils during the early stages of SS could have difficulties mounting effective innate responses against the invading microbe(s). Increased neutrophil adhesion to the vascular endothelium in sepsis could contribute to lower CNC. Neutrophils adhered to the blood vessel wall seem to induce endothelial damage [4], forming leukocyte aggregates that could lead to microvascular thrombosis $[1,5]$. Host immunity compromise and/or increased endothelial damage could both impair outcome in these patients.

$\mathrm{CNC}$ at diagnosis is a major prognostic factor in SS. Our work provides a CNC cutoff that is potentially useful as a prognostic indicator.

\section{Abbreviations}

CNC: Circulating neutrophil count; SS: Septic shock.

\section{Competing interests}

The authors declare that they have no competing interests.

\section{Authors' contributions}

JFBM, GR, DAO, and MFM designed the study, analyzed the data and participated in writing the article; ET, JB, and JIGH helped with the study design, provided a critical review of the results and participated in writing the article; RHM, AMB, MHR, and RC provided a critical review of the results and participated in writing the article. All authors have read and approved the final version for publication.

\footnotetext{
* Correspondence: jfbermejo@saludcastillayleon.es

${ }^{\dagger}$ Equal contributors

'Unidad de Investigación Biomédica, Hospital Clínico Universitario de Valladolid, SACYL/IECSYL, Avda Ramón y Cajal 3, 47005 Valladolid, Spain Full list of author information is available at the end of the article
} 
Table 1 Clinical characteristics of the patients in the discovery study in survivors and non-survivors at 28 days

\begin{tabular}{|c|c|c|c|c|}
\hline & Total $(n=195)$ & Survivors $(n=125)$ & Non-survivors $(n=70)$ & $P$ \\
\hline \multicolumn{5}{|l|}{ Patient details } \\
\hline Gender (male) & $125(64.1 \%)$ & $79(63.2 \%)$ & $46(65.7 \%)$ & NS \\
\hline Age (years) & $71.6 \pm 11.1$ & $70.2 \pm 11.1$ & $74.2 \pm 10.7$ & 0.014 \\
\hline Hypertension & 109 (55.9\%) & $71(56.8 \%)$ & $38(54.3 \%)$ & NS \\
\hline Cardiovascular disease & 87 (44.6\%) & $54(43.2 \%)$ & $33(47.1 \%)$ & NS \\
\hline Cancer & $44(22.5 \%)$ & $21(16.8 \%)$ & $23(32.8 \%)$ & 0.010 \\
\hline COPD & $33(16.9 \%)$ & $24(19.2 \%)$ & $9(12.8 \%)$ & NS \\
\hline Diabetes & $31(15.9 \%)$ & $24(19.2 \%)$ & $7(10 \%)$ & NS \\
\hline Obesity & $32(16.4 \%)$ & $21(16.8 \%)$ & $11(15.7 \%)$ & NS \\
\hline Smoker & $27(13.8 \%)$ & $17(13.6 \%)$ & $10(14.2 \%)$ & NS \\
\hline Chronic renal failure & $26(13.3 \%)$ & $15(12.0 \%)$ & $11(15.7 \%)$ & NS \\
\hline Alcohol abuse & $12(6.1 \%)$ & $8(6.4 \%)$ & $4(5.7 \%)$ & NS \\
\hline Inmunosuppression & $9(4.6 \%)$ & $5(4.0 \%)$ & $4(5.7 \%)$ & NS \\
\hline Hepatic disease & $6(3.1 \%)$ & $4(3.2 \%)$ & $2(2.8 \%)$ & NS \\
\hline \multicolumn{5}{|l|}{ Clinical status at admission } \\
\hline APACHE ॥ & $14.7 \pm 5.9$ & $13.9 \pm 5.8$ & $16.2 \pm 5.9$ & 0.013 \\
\hline Mechanical ventilation & $134(68.7 \%)$ & $83(66.4 \%)$ & $51(72.9 \%)$ & NS \\
\hline OARF & $41(21.0 \%)$ & $19(15.2 \%)$ & $22(31.4 \%)$ & 0.008 \\
\hline \multicolumn{5}{|l|}{ Presumed source of infection } \\
\hline Digestive system & $115(58.9 \%)$ & $76(60.8 \%)$ & $39(55.7 \%)$ & NS \\
\hline Respiratory system & $19(9.7 \%)$ & $14(11.2 \%)$ & $5(7.1 \%)$ & NS \\
\hline Central nervous system & $20(10.2 \%)$ & $14(11.2 \%)$ & $6(8.5 \%)$ & NS \\
\hline Urinary system & $10(5.1 \%)$ & $5(4.0 \%)$ & $5(7.1 \%)$ & NS \\
\hline Endocardium & 7 (3.5\%) & $5(4.0 \%)$ & $2(2.8 \%)$ & NS \\
\hline Catheter & $34(17.4 \%)$ & $26(20.8 \%)$ & $8(11.4 \%)$ & NS \\
\hline Wound/skin, soft tissue & $28(14.3 \%)$ & $20(16.0 \%)$ & $8(11.4 \%)$ & NS \\
\hline Other/unknown & $55(28.2 \%)$ & $35(28.0 \%)$ & $20(28.5 \%)$ & NS \\
\hline \multicolumn{5}{|l|}{ Type of surgery } \\
\hline Abdominal & $99(50.7 \%)$ & $56(44.8 \%)$ & $43(61.4 \%)$ & 0.030 \\
\hline Cardiac & $71(36.4 \%)$ & $54(43.2 \%)$ & $17(24.3 \%)$ & \\
\hline Other & $25(12.8 \%)$ & $15(12.0 \%)$ & $10(14.3 \%)$ & \\
\hline \multicolumn{5}{|l|}{ Urgent surgery } \\
\hline Yes & $130(66.6 \%)$ & $77(61.6) \%$ & $53(75.7 \%)$ & 0.045 \\
\hline \multicolumn{5}{|l|}{ Documented microbial agent } \\
\hline Gram-negative & $67(41.6 \%)$ & $48(44.4 \%)$ & $19(35.8 \%)$ & NS \\
\hline Gram-positive & $68(42.2 \%)$ & $47(43.5 \%)$ & $21(39.6 \%)$ & NS \\
\hline Fungi & $24(14.9 \%)$ & $16(14.8 \%)$ & $8(15.1 \%)$ & NS \\
\hline \multicolumn{5}{|l|}{ Laboratory data } \\
\hline Bilirubin (mg/dL) & $1.4 \pm 1.3$ & $1.5 \pm 1.4$ & $1.2 \pm 1.0$ & NS \\
\hline Glycemia (mg/dL) & $166.4 \pm 65.1$ & $165.4 \pm 58.3$ & $168.2 \pm 76.0$ & NS \\
\hline Procalcitonin (ng/mL) & $19.3 \pm 32.5$ & $16.5 \pm 28.6$ & $24.3 \pm 38.1$ & NS \\
\hline $\mathrm{CRP}(\mathrm{mg} / \mathrm{mL})$ & $231.8 \pm 119.2$ & $221.9 \pm 106.6$ & $249.4 \pm 138.1$ & NS \\
\hline INR & $1.7 \pm 0.9$ & $1.6 \pm 0.9$ & $1.7 \pm 0.8$ & NS \\
\hline Platelets $\left(\times 10^{3} / \mu \mathrm{l}\right)$ & $190.2 \pm 140.0$ & $196.9 \pm 143.5$ & $178.2 \pm 133.5$ & NS \\
\hline Leukocytes $\left(\times 10^{3} / \mu \mathrm{l}\right)$ & $16.3 \pm 10.1$ & $16.4 \pm 9.0$ & $16.2 \pm 11.7$ & NS \\
\hline Monocytes $\left(\times 10^{3} / \mu \mathrm{l}\right)$ & $0.7 \pm 0.4$ & $0.7 \pm 0.4$ & $0.6 \pm 0.5$ & NS \\
\hline Lymphocyte $\left(\times 10^{3} / \mu \mathrm{l}\right)$ & $1.1 \pm 0.7$ & $1.1 \pm 0.8$ & $1.0 \pm 0.6$ & NS \\
\hline Neutrophils $\left(\times 10^{3} / \mu \mathrm{l}\right)$ & $14.4 \pm 9.4$ & $14.4 \pm 8.4$ & $14.3 \pm 11.1$ & NS \\
\hline Basophils $\left(\times 10^{3} / \mu \mathrm{l}\right)$ & $0.1 \pm 0.0$ & $0.1 \pm 0.1$ & $0.1 \pm 0.0$ & NS \\
\hline Eosinophils $\left(\times 10^{3} / \mu \mathrm{l}\right)$ & $0.1 \pm 0.0$ & $0.1 \pm 0.0$ & $0.1 \pm 0.0$ & NS \\
\hline
\end{tabular}

For the demographic characteristics of the patients, differences between groups were assessed using the X2 test for categorical variables and the Student's $t$-test for continuous variables when appropriate. Continuous variables are expressed as mean \pm standard deviation. APACHE, Acute Physiology and Chronic Health Evaluation; COPD, chronic obstructive pulmonary disease; CRP, C reactive protein; INR, international normalized ratio; NS, not significant; OARF, oliguric acute renal failure. 
Table 2 Clinical characteristics of the patients in the validation study in survivors and non-survivors at 28 days

\begin{tabular}{|c|c|c|c|c|}
\hline & Total $(n=194)$ & Survivors $(n=132)$ & Non-survivors $(n=62)$ & $P$ \\
\hline \multicolumn{5}{|l|}{ Patient details } \\
\hline Gender (male) & $126(64.9 \%)$ & $85(64.3 \%)$ & $41(66.1 \%)$ & NS \\
\hline Age (years) & $67.1 \pm 13.3$ & $65.3 \pm 14.3$ & $71.1 \pm 9.5$ & $<0.001$ \\
\hline Inmunosuppression & $35(18.0 \%)$ & $15(11.3 \%)$ & $20(32.2 \%)$ & $<0.001$ \\
\hline Diabetes & $32(16.4 \%)$ & $21(15.9 \%)$ & $11(17.7 \%)$ & NS \\
\hline Cardiovascular disease & $24(12.3 \%)$ & $14(10.6 \%)$ & $10(16.1 \%)$ & NS \\
\hline Cancer & $18(9.2 \%)$ & $10(7.5 \%)$ & $8(12.9 \%)$ & NS \\
\hline COPD & $23(11.8 \%)$ & $12(9.0 \%)$ & $11(17.7 \%)$ & NS \\
\hline Chronic renal failure & $15(7.7 \%)$ & $10(7.5 \%)$ & $5(8.0 \%)$ & NS \\
\hline Alcohol abuse & $12(6.1 \%)$ & $7(5.3 \%)$ & $5(8.0 \%)$ & NS \\
\hline Hepatic disease & $4(2.0 \%)$ & $1(0.7 \%)$ & $3(4.8 \%)$ & NS \\
\hline \multicolumn{5}{|l|}{ Clinical status at admission } \\
\hline APACHE II score & $22.6 \pm 7.0$ & $21.0 \pm 6.5$ & $25.9 \pm 7.1$ & $<0.001$ \\
\hline Mechanical ventilation & $150(77.7 \%)$ & 93 (70.9\%) & 57 (91.9\%) & $<0.001$ \\
\hline OARF & $39(20.1 \%)$ & $17(12.8 \%)$ & $22(35.4 \%)$ & $<0.001$ \\
\hline \multicolumn{5}{|l|}{ Presumed source of infection } \\
\hline Respiratory system & 67 (34.5\%) & $45(34.1 \%)$ & $22(35.5 \%)$ & NS \\
\hline Digestive system & $52(26.8 \%)$ & $32(24.2 \%)$ & $20(32.3 \%)$ & NS \\
\hline Urinary system & $26(13.4 \%)$ & $21(15.9 \%)$ & $5(8.1 \%)$ & NS \\
\hline Catheter & $16(8.2 \%)$ & $11(8.3 \%)$ & $5(8.1 \%)$ & NS \\
\hline Wound/skin, soft tissue & $15(7.7 \%)$ & $11(8.3 \%)$ & $4(6.5 \%)$ & NS \\
\hline Other/unknown & $18(9.3 \%)$ & $12(9.1 \%)$ & $6(9.7 \%)$ & NS \\
\hline \multicolumn{5}{|l|}{ Documented microbial agent } \\
\hline Gram-negative & $52(26.8 \%)$ & $36(27.2 \%)$ & $16(25.8 \%)$ & NS \\
\hline Gram-positive & $33(17.0 \%)$ & $25(18.9 \%)$ & $8(12.9 \%)$ & NS \\
\hline Fungi & $12(6.1 \%)$ & $4(3.0 \%)$ & $8(12.9 \%)$ & 0.020 \\
\hline \multicolumn{5}{|l|}{ Laboratory data } \\
\hline Bilirubin (mg/dL) & $1.4 \pm 2.2$ & $1.4 \pm 2.1$ & $1.6 \pm 2.4$ & NS \\
\hline Glycemia (mg/dL) & $168 \pm 64.0$ & $167 \pm 62.4$ & $172.0 \pm 67.6$ & NS \\
\hline INR & $1.8 \pm 3.1$ & $1.9 \pm 3.8$ & $1.6 \pm 0.6$ & NS \\
\hline Platelets $\left(\times 10^{3} / \mu\right.$ l) & $177.4 \pm 118.5$ & $173.6 \pm 105.2$ & $186.4 \pm 146.1$ & NS \\
\hline Leukocytes $\left(\times 10^{3} / \mu l\right)$ & $18.0 \pm 16.4$ & $18.4 \pm 17.0$ & $17.3 \pm 15.3$ & NS \\
\hline Monocytes $\left(\times 10^{3} / \mu \mathrm{l}\right)$ & $0.7 \pm 1.7$ & $0.8 \pm 20.2$ & $0.6 \pm 0.9$ & NS \\
\hline Lymphocyte $\left(\times 10^{3} / \mu l\right)$ & $1.8 \pm 70.5$ & $1.6 \pm 73.5$ & $2.3 \pm 64.1$ & NS \\
\hline Neutrophils $\left(\times 10^{3} / \mu \mathrm{l}\right)$ & $14.9 \pm 12.5$ & $15.6 \pm 12.9$ & $13.6 \pm 11.5$ & NS \\
\hline Basophils $\left(\times 10^{3} / \mu \mathrm{l}\right)$ & $0.1 \pm 0.0$ & $0.1 \pm 0.0$ & $0.1 \pm 0.0$ & NS \\
\hline Eosinophils $\left(\times 10^{3} / \mu \mathrm{l}\right)$ & $0.1 \pm 0.0$ & $0.1 \pm 0.0$ & $0.1 \pm 0.0$ & NS \\
\hline
\end{tabular}

For the demographic characteristics of the patients, differences between groups were assessed using the $\mathrm{X} 2$ test for categorical variables and the Student's $t$-test for continuous variables when appropriate. Continuous variables are expressed as mean \pm standard deviation. APACHE, Acute Physiology and Chronic Health Evaluation; COPD, chronic obstructive pulmonary disease; INR, international normalized ratio; NS, not significant; OARF, oliguric acute renal failure. 


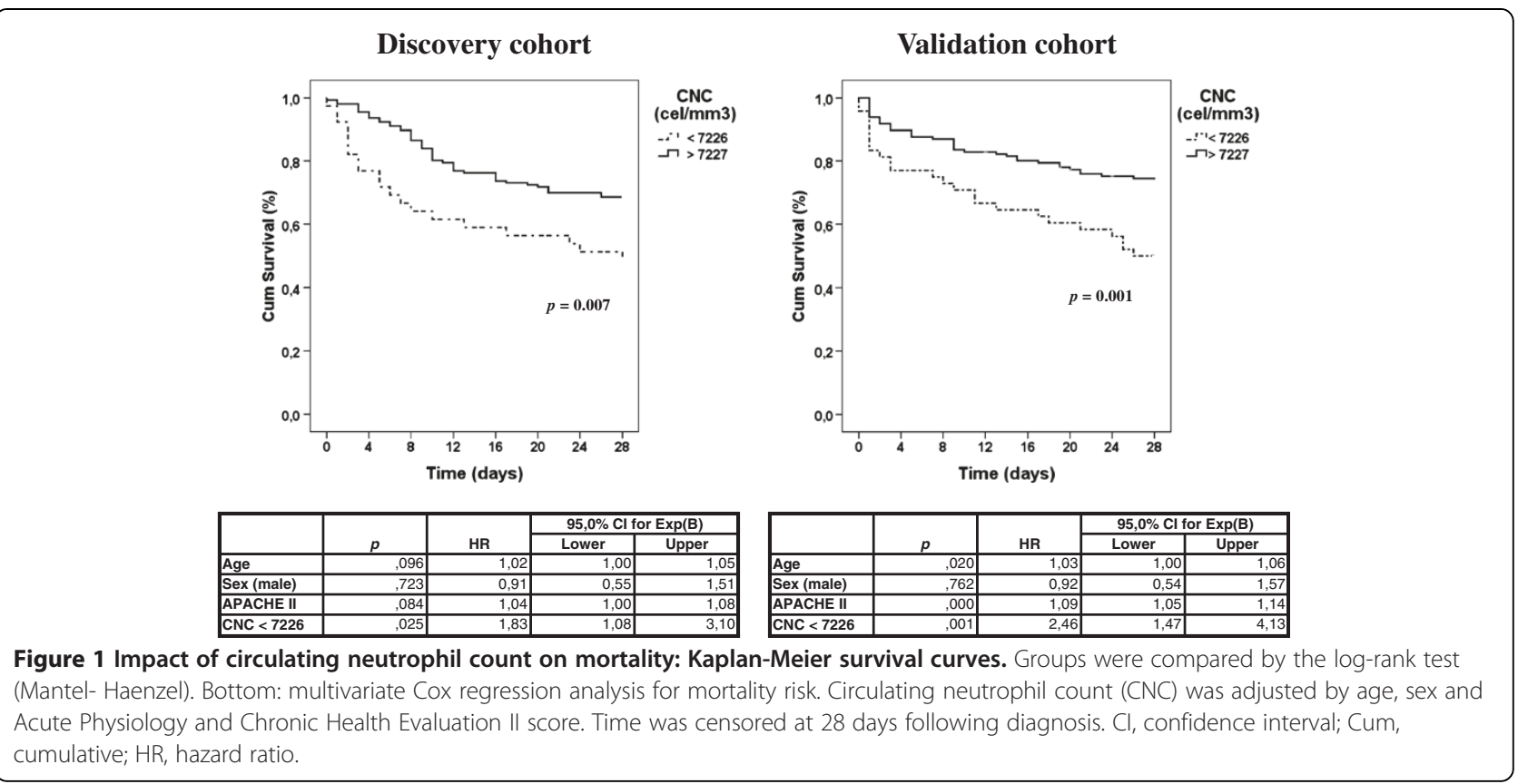

\section{Acknowledgements}

Discovery study (EXPRESS) was supported by ISCIII, 'Proyectos de Investigación Sanitaria, PI 10/01362. Validation study (GRECIA) was supported by Proyectos de investigación en Biomedicina, Health Council, Castilla y León Goverment, Spain (BOCYL-D-26072010). Investigators of the EXPRESS group: Esther Gómez-Sánchez, Sandra Gutiérrez, Felipe Bobillo, Francisco Gandía, Verónica Iglesias , Lucia Rico, Raquel Almansa, Raúl Ortiz de Lejarazu (Hospital Clínico Universitario, Valladolid, Spain). Investigators of the GRECIA group: Pedro Merino, Marta María García-García (Hospital Universitario Río Hortega, Valladolid, Spain); Ma Jesús López Pueyo, Jose Antonio Fernandez Ratero, Miguel Martinez Barrios, Fernando Callejo Torre, Sergio Ossa Echeverri (Hospital General Yagüe, Burgos, Spain); Demetrio Carriedo Ule, Ana Ma Domínguez Berrot, Fco Javier Díaz Domínguez (Complejo Hospitalario de León, Spain); Susana Moradillo (Hospital Río Carrión, Palencia, Spain); Braulio Alvarez Martínez (Hospital del Bierzo, Ponferrada, Spain); Noelia Albalá, Juan Carlos Ballesteros, Marta Paz Perez, Elena Perez Losada (Hospital Clínico Universitario de Salamanca, Spain); Santiago Macías, Rafael Pajares García, Noelia Recio García Cervigón (Hospital General de Segovia Spain); Ma Mar Gobernado Serrano, Ma José Fernández Calavia, Daniel Moreno Torres (Complejo Hospitalario de Soria, Spain); Concha Tarancón, Teresa Loreto, Priscila Carcelen (Hospital Virgen de la Concha, Spain).

\section{Author details}

${ }^{1}$ Unidad de Investigación Biomédica, Hospital Clínico Universitario de Valladolid, SACYL/IECSYL, Avda Ramón y Cajal 3, 47005 Valladolid, Spain ${ }^{2}$ Servicio de Anestesiología, Hospital Clínico Universitario de Valladolid, SACYL, Avda Ramón y Cajal 3, 47005 Valladolid, Spain. ${ }^{3}$ Servicio de Medicina Preventiva, Hospital Clínico Universitario de Valladolid, SACYL, Avda Ramón y Cajal 3, 47005 Valladolid, Spain. ${ }^{4}$ Servicio de Medicina Intensiva, Hospital Clínico Universitario de Valladolid, SACYL, Avda Ramón y Cajal 3, 47005 Valladolid, Spain. ${ }^{5}$ Servicio de Medicina Intensiva, Hospital Universitario Río Hortega, SACYL, Calle Dulzania, 2, 47012 Valladolid, Spain. ${ }^{6}$ Centro de investigación en red de enfermedades respiratorias (CIBERES), Hospital Universitario Río Hortege, Calle Dulzania, 2, 47012 Valladoid, Spain.

\section{Published: 14 February 2014}

\section{References}

1. Brown KA, Brain SD, Pearson JD, Edgeworth JD, Lewis SM, Treacher DF: Neutrophils in development of multiple organ failure in sepsis. Lancet 2006, 368:157-169.

2. Bone RC, Balk RA, Cerra FB, Dellinger RP, Fein AM, Knaus WA, Schein RM, Sibbald WJ, ACCP/SCCM Consensus Conference Committee: Definitions for sepsis and organ failure and guidelines for the use of innovative therapies in sepsis. The ACCP/SCCM Consensus Conference Committee. American College of Chest Physicians/Society of Critical Care Medicine. 1992. Chest 2009, 136:e28.

3. Lim E-M, Cembrowski G, Cembrowski M, Clarke G: Race-specific WBC and neutrophil count reference intervals. Int I Lab Hematol 2010, 32:590-597.

4. Fox ED, Heffernan DS, Cioffi WG, Reichner JS: Neutrophils from critically ill septic patients mediate profound loss of endothelial barrier integrity. Crit Care 2013, 17:R226.

5. Brown KA, Treacher DF: Neutrophils as potential therapeutic targets in sepsis. Discov Med 2006, 6:118-122.

doi:10.1186/cc13728

Cite this article as: Bermejo-Martín et al:: Circulating neutrophil counts and mortality in septic shock. Critical Care 2014 18:407. 